\title{
Chronic Non-Communicable Diseases, a major challenge facing contemporary society
}

The Revista Ciência e Saúde Coletiva journal dedicates this edition to "Diseases and Complications Associated with Lifestyle," and addresses the complexity of Chronic Non-Communicable Diseases (NCDs) in the twenty-first century. NCDs, including cardiovascular disease, cancer, diabetes and chronic respiratory diseases have generated a high number of premature deaths, loss of quality of life, and caused negative economic impacts for families, individuals and society in general. They are now responsible for $72 \%$ of the mortality in Brazil and are more prevalent among the poor, because they are more exposed to risk factors and have less access to health services. The increased burden of NCDs reflects the negative effects of globalization, rapid urbanization, sedentary life and food with high calorie levels and marketing that encourages the use of tobacco and alcohol.

Stemming the increase of NCDs is a challenge that led the United Nations to convene a high-level meeting in 2011, which resulted in a comprehensive plan for tackling the issue approved by more than 190 member countries in 2013 May. This commitment was made by Brazil, which launched the Strategic Action Plan for Tackling NCDs 2011-2022, with goals for reducing mortality and risk factors. The drop in NCD rates requires the commitment of governments, the public and private sectors and society as a whole. It cannot be treated only as an individual option for healthy choices.

In 2011, the World Health Organization divulged interventions considered more cost-effective in the protection against NCDs and recommended that they be implemented immediately in order to produce results that save lives, prevent disease and reduce social costs. Among the measures, the highlights are an increase in taxes and the price of tobacco, creating smoke-free environments, warnings about the dangers of consumption of this product and on the advertising for it. The majority of these measures have already been implemented in Brazil and resulted in one of the greatest reductions in global tobacco consumption. The country had $34.8 \%$ of smokers in 1989, which dropped to $12.1 \%$ in 2012, according to the latest research by Vigitel in the state capitals. Preventive measures against alcohol abuse include restricting retail sales, banning sale to minors and in the application of the law concerning the prohibition of alcohol to anyone who drives a motor vehicle. With respect to food, there are measures to reduce salt in food, eliminate unsaturated fats (trans fats), promote adequate breastfeeding and implement restrictions on the marketing of food products, especially aimed at children.

The WHO stresses the importance of investing in communication about food products and physical activity and the promotion of physical activity programs and healthy eating in schools, workplaces and community areas. It also encourages investments in healthy spaces, urban mobility in a proactive manner, including bicycle paths, places for hiking and walking, physical exercise and public transportation.

Therefore, preventing NCDs implies acting on regulatory measures for tobacco, alcohol and food products, building healthy urban spaces and investment in public policies aimed at collective protection. In other words, it requires decisions involving the public authorities, though mainly the adherence and mobilization of society, seeking new sustainable and healthy lifestyle prospects

In order to tackle NCDs, besides the mobilization of the health sector to ensure access to health, promotion, prevention and surveillance, it is essential to articulate intersectoral actions, especially those that contribute to reducing social inequalities and protecting the most vulnerable sectors of the population, especially children and adolescents. Policies to tackle NCDs should be coordinated, integrated and cooperative.

Deborah Carvalho Malta

Diretora de Vigilância de Doenças e Agravos Não Transmissíveis e Promoção da Saúde, Secretaria de Vigilância em Saúde do Ministério da Saúde 